11

\title{
Оптоакустическое исследование и моделирование оптических свойств композитов циклотриметилентринитрамин-ультрадисперсные частицы никеля
}

\author{
(C) Б.П. Адуев ${ }^{1}$, Д.Р. Нурмухаметов ${ }^{1}$, А.А. Звеков ${ }^{1}$, Н.В. Нелюбина ${ }^{1}$, С.А. Созинов ${ }^{1}$, \\ А.В. Каленский ${ }^{2,}$, М.В. Ананьева ${ }^{2}$, Е.В. Галкина ${ }^{2}$ \\ ${ }^{1}$ Институт углехимии и химического материаловедения \\ Федерального исследовательского центра угля и углехимии Сибирского отделения РАН, \\ 650000 Кемерово, Россия \\ ${ }^{2}$ Кемеровский государственный университет, \\ 650000 Кемерово, Россия \\ ؟e-mail: kriger@kemsu.ru
}

Поступила в редакцию 20.11.2019 г.

В окончательной редакции 27.12.2019 г.

Принята к публикации 13.01.2020 г.

\begin{abstract}
Экспериментально получены оптоакустические сигналы, инициируемые импульсным лазерным излучением (длительность импульса на полувысоте $14 \mathrm{~ns}$ ) с длиной волны $532 \mathrm{~nm}$ в прессованных композитах циклотриметилентринитрамин (RDX) -ультрадисперсные частицы никеля, и определены их наблюдаемые показатели поглощения излучения при значениях среднего радиуса частиц 40, 60, 80 и $140 \mathrm{~nm}$. Показано, что зависимость экспериментального показателя поглощения от радиуса частиц при постоянной массовой доле немонотонная. Результаты интерпретировались в рамках теории переноса монохроматического излучения. Показано, что согласие экспериментальных и рассчитанных значений показателя поглощения прессованных таблеток RDX-ультрадисперсные частицы никеля различного радиуса достигается при учете остаточной пористости образца. Оценены средний радиус пор и их концентрация.
\end{abstract}

Ключевые слова: оптоакустическая спектроскопия, ультрадисперсные частицы металлов, RDX, уравнение переноса излучения.

DOI: $10.21883 /$ OS.2020.05.49327.313-19

\section{Введение}

Исследование оптических свойств наноразмерных систем является одной из основных задач современной оптики и спектроскопии. В работах последнего десятилетия акцент сместился от рассмотрения систем, состоящих из отдельных однородных наночастиц шарообразной формы, к исследованию эллипсоидов $[1,2]$, цилиндров [2,3], прямоугольных параллелепипедов [4], димеров и тримеров $[1,5]$, цепочек наночастиц [6-8], двух[5,9-11] и трехслойных частиц типа ядро-оболочка [12]. Сформулированы и апробированы подходы к моделированию спектральных свойств систем, содержащих частицы со сложной структурой $[1,2,5-9,12]$.

Оптические характеристики ультрадисперсных частиц существенно изменяются при получении наноматериалов, поэтому возникает задача их определения в исполнительных устройствах на основе измеряемых оптических свойств функциональных материалов. В работах $[13,14]$ предложены методы обработки результатов измерений оптических свойств систем, состоящих из прозрачной матрицы и частиц металлов, отстоящих друг от друга на расстояние, значительно превышающее длину волны, с использованием подходов диффузного отражения и пропускания, которые позволяют опреде- лить параметры содержащихся в них частиц. Схема использованного алгоритма приведена на рис. 1. Блок 1 включает формулировку модели процесса рассеяния и поглощения излучения ультрадисперсными частицами, которая учитывает форму и природу частиц, однородность или структурированность, оптические константы материалов и длину волны излучения. В блоке 2 используется теория Ми и ее модификации для расчета оптических свойств частиц, которые зависят от значений комплексного показателя преломления металла и показателя преломления матрицы при используемых длинах волны и температуре [14-16]. В ряде случаев добавляется блок 2a, в котором учитывается распределение геометрических параметров наносистемы, например, радиусов частиц или толщины диэлектрической оболочки $[13,15]$. В блоке 3 проводится вычисление показателей поглощения и рассеяния элементарного объема среды с учетом концентрации частиц, а также фазовой функции и среднего косинуса угла рассеяния. В блоке 4 моделируется процесс переноса излучения в рассматриваемом образце, и рассчитываются его оптические свойства с учетом многократного рассеяния света: коэффициенты коллимированного и диффузного пропускания и отражения, распределение поглощенной мощности по глубине образца. Если в качестве экс- 


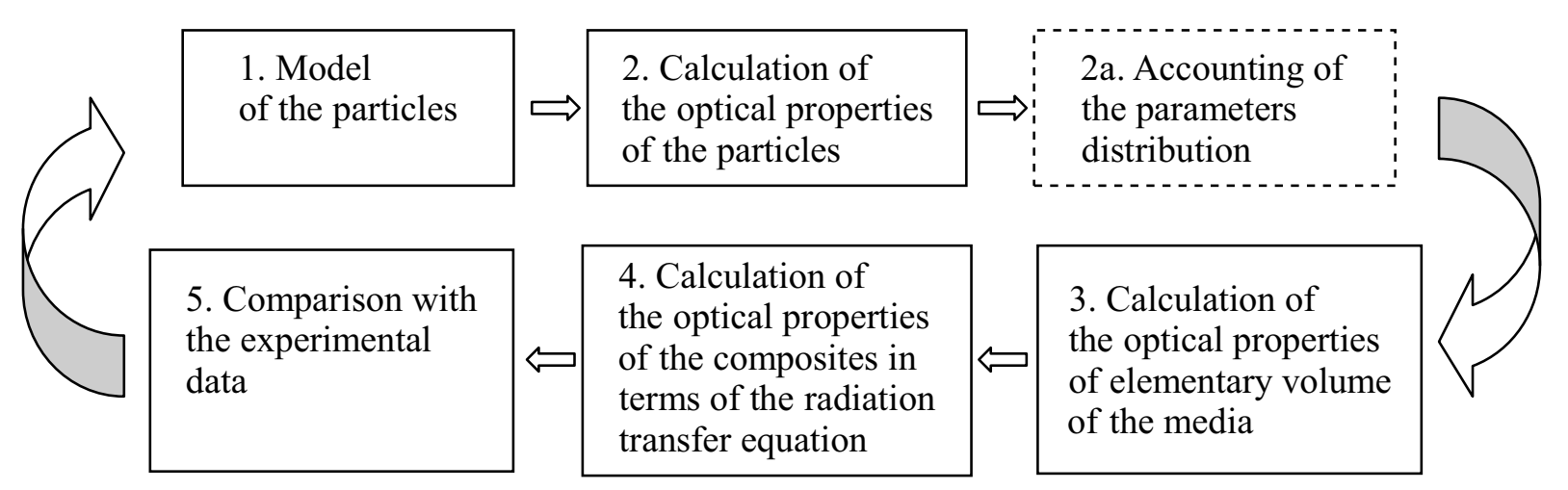

Рис. 1. Схема алгоритма определения оптических свойств наночастиц на основе результатов измерений оптических характеристик композита.

периментальных данных для решения обратной задачи используются только значения коэффициента коллимированного пропускания света, блок 4 не является обязательным [16]. В блоке 5 проводится сравнение результатов эксперимента и расчета, на основании которого корректируются параметры модели процесса переноса излучения, например, характерные размеры частицы или комплексный показатель преломления материала ультрадисперсных частиц $[13,16]$. Цикл повторяется до достижения удовлетворительного согласия результатов расчета и эксперимента. Алгоритм может быть реализован сразу для нескольких длин волн [17] или широкого спектрального диапазона [16].

Значительная часть элементов данного алгоритма использовалась при моделировании оптических свойств снега с вкраплениями сажи [18], композитов пентаэритрит тетранитрат (РETN)-наночастицы алюминия [13] и циклотриметилентринитрамин (RDX)-наночастицы алюминия типа металлическое ядро-оксидная оболочка [14] и металлическое ядро-воздушная оболочка, связанная с остаточной пористостью материала [15]. Таким образом, данная методика обеспечивает решение прямой и обратной задач спектроскопии систем, содержащих частицы металлов в непоглощающей свет матрице, на количественном уровне.

В процитированных работах в качестве экспериментальных данных использовались результаты фотометрических измерений коэффициентов пропускания и отражения. В случае образцов с большой оптической плотностью более удобными становятся фототермические методы исследования, такие как термическое зеркало [19] и оптоакустическая спектроскопия [20-22]. В работе [23] предложена методика моделирования оптоакустических сигналов, инициируемых лазерным импульсом в поглощающей среде с упругим рассеянием излучения. Цель настоящей работы: экспериментальное исследование оптических свойств композитов RDX-ультрадисперсные частицы никеля методами оптоакустической спектроскопии с определением особенностей поглощения и рассеяния света в данной системе.
Композиционный материал RDX-никель выбран в качестве объекта исследования, так как ранее показана перспективность использования подобных систем в качестве капсульных составов оптических детонаторов [24]. Оптические свойства композитов RDX-алюминий были ранее исследованы в [15]. Введение частиц в материал повышает его способность к поглощению электромагнитного излучения и таким образом сенсибилизирует лабильную матрицу. Ультрадисперсные частицы никеля лучше поглощают излучение основной и второй гармоник неодимового лазера $[25,26]$, что позволяет предположить наличие у состава RDX-никель чувствительности к воздействию лазерного импульса, сопоставимой с чувствительностью, полученной для составов RDXалюминий.

Оптоакустическая спектроскопия является общепринятым способом исследования оптических свойств материалов. Она показала свою применимость при измерении малых концентраций газов в воздухе [27,28], определении оптических свойств материалов с сильным поглощением света $[20,29]$ и высокопористых образцов [30]. В биомедицинской области развиваются методы оптоакустической томографии для диагностики онкологических заболеваний [31].

\section{Методика эксперимента}

Экспериментальные образцы представляли собой прессованные таблетки на основе RDX с добавками ультрадисперсных частиц никеля. Использовался порошок RDX с узким гранулометрическим распределением, в максимуме распределения размер составляет $1-2 \mu \mathrm{m}[14]$.

Ультрадисперсный порошок никеля был получен Р.П. Колмыковым восстановлением хлорида никеля гидразингидратом в водном растворе [32] и характеризуется очень широким распределением по размерам. Для выделения фракций с различными средними радиусами и более узким распределением использовалась седиментационная техника в водной среде в поле силы тяжести. 

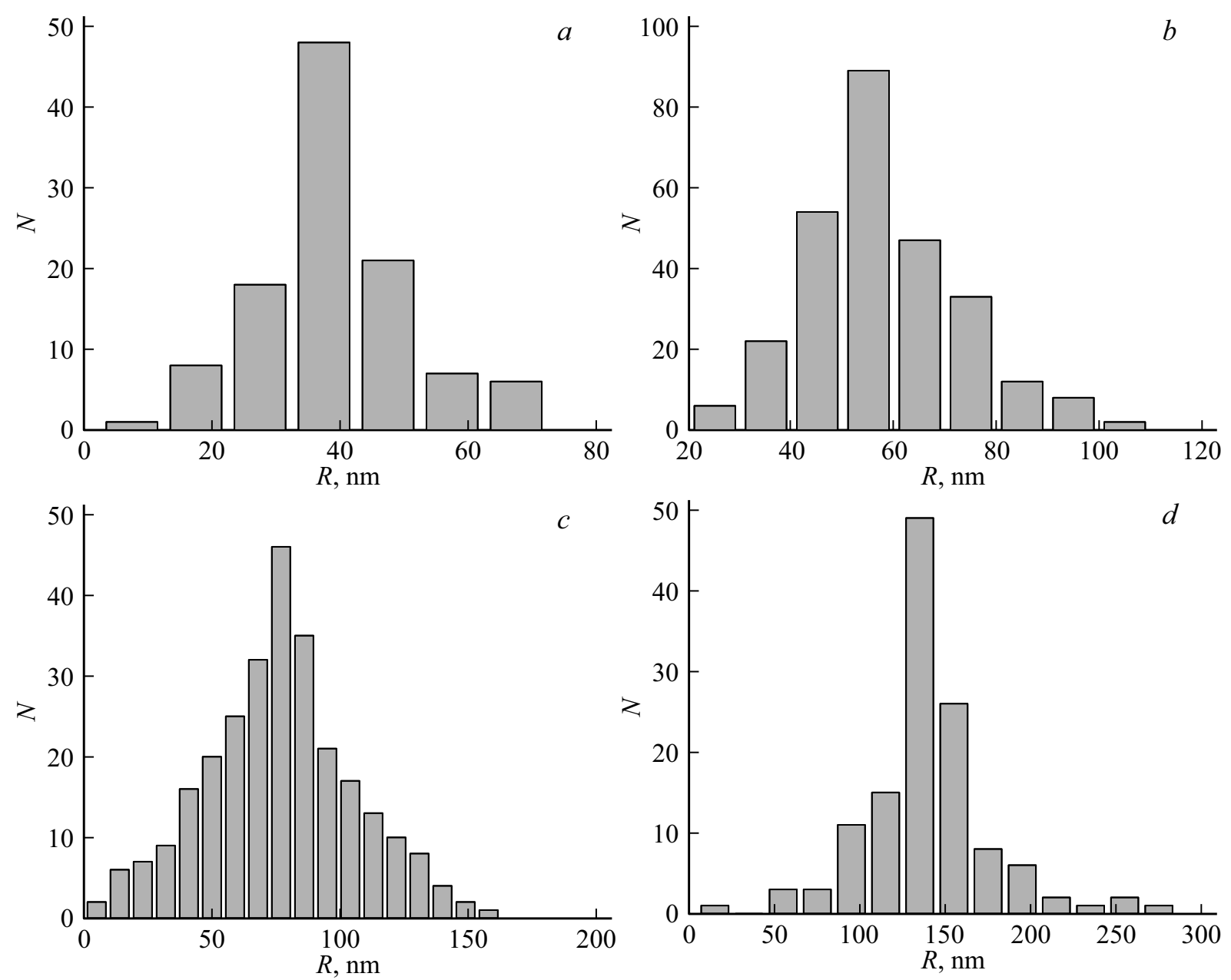

Рис. 2. Распределения частиц никеля по радиусам в образцах со средними радиусами $40(a), 60(b), 80(c), 140 \mathrm{~nm}(d)$, использованных для приготовления прессованных композитов RDX-Ni.

В результате варьирования условий были получены 4 партии порошков ультрадисперсных частиц никеля со средними радиусами 40, 60, 80 и $140 \mathrm{~nm}$. Гистограммы распределения частиц по радиусам приведены на рис. 2.

Экспериментальные образцы готовились следующим образом. В порошок RDX добавлялся порошок ультрадисперсных частиц никеля до получения нужной массовой доли. Смесь помещалась в четыреххлористый углерод и перемешивалась в ультразвуковой ванне для получения равномерного распределения частиц в объеме смеси. После этого проводили испарение четыреххлористого углерода, сушку смеси и отбирали навеску образца, значение которой составляло $12.7 \pm 0.3 \mathrm{mg}$. Образцы прессовались с применением пресс-формы в медной пластине толщиной $1 \mathrm{~mm}$, в центре которой имелось отверстие диаметром $3 \mathrm{~mm}$ с массовой долей наночастиц $0.05 \%$. При прессовании давление поднималось в течение $30 \mathrm{~min}$ до значения $1.8 \mathrm{GPa}$. В результате прессования в центре пластины получали образец с плотностью, близкой к плотности монокристалла $\left(1.80 \pm 0.02 \mathrm{~g} / \mathrm{cm}^{3}\right)$, диаметром $3 \mathrm{~mm}$ и толщиной $1 \mathrm{~mm}$. Методика приготовления образцов аналогична используемой в [14] для приготовления прессованных таблеток RDX-ультрадисперсные частицы алюминия и PETN-частицы алюминия, никеля или железа [13,24,25].

Для возбуждения оптоакустических сигналов использовалось излучение импульсного YAG: $\mathrm{Nd}^{3+}$-лазеpa LQ929 (SOLAR Laser Systems, Минск), работающего в режиме модуляции добротности. Длительность импульса $\tau_{i}$ составляла $14 \mathrm{~ns}$. Использовалось излучение второй $(\lambda=532 \mathrm{~nm})$ гармоники лазера. Плотность энергии в импульсе составляла $30 \mathrm{~mJ} / \mathrm{cm}^{2}$. Выбранное значение плотности энергии, во-первых, позволяет получать надежно измеряемые оптоакустические сигналы, а во-вторых, является достаточно малым и не вызывает повреждения образцов. Измерение оптоакустических (ОА) сигналов импульсным ОА методом проводилось по схеме с прямой регистрацией [33] (рис. 3). Основная часть излучения фокусировалась линзой 3 на образец 4 в медной пластине. Диаметр лазерного пучка на поверхности образца составлял $2 \mathrm{~mm}$ с однородным распределением интенсивности. Образец находился в акустическом контакте через дюралюминиевую акустическую задержку 5 с пьезопреобразователем 6, изготовленным 
из керамики ЦТС-19 (диаметром $8 \mathrm{~mm}$, толщиной $4 \mathrm{~mm}$, с напыленными серебряными электрическими контактами). Часть энергии лазерного излучения светоделительной пластиной 7 отводилась на калиброванный фотодиод для контроля энергии импульса. Энергия лазерного излучения определялась с помощью пироэлектрического измерителя энергии PE50BF-C (Ophir ${ }^{\circledR}$ Photonics). Нестабильность энергии инициирующего импульса не превышала $3 \%$. Воздействие излучения производилось на открытую поверхность образцов. Сигнал с пьезопреобразователя регистрировался цифровым осциллографом 9 LeCroy WJ332A (полоса пропускания $350 \mathrm{MHz}$, частота дискретизации $2 \mathrm{GHz}$ ). Синхронизация элементов установки осуществлялась внутренним генератором блока управления лазера.

\section{Результаты эксперимента}

На рис. 4, а представлены начальные участки экспериментальных оптоакустических сигналов, инициируемых излучением второй гармоники импульсного неодимового лазера в прессованных композитах RDX-ультрадисперсные частицы никеля, которые отражают распределение тепловых источников в среде [20]. Все зависимости на начальном участке от 5 до $50 \%$ амплитуды могут быть удовлетворительно описаны экспоненциальными функциями (рис. $4, b$ ). Из сравнения зависимостей следует, что эффективные константы нарастания сигнала, равные коэффициенту наклона прямой в полулогарифмических координатах, отличаются, составляя 18.7, 22.2, 17.3 и $9.9 \mu \mathrm{s}^{-1}$ для добавок ультрадисперсных частиц никеля со средним радиусом 40, 60, 80 и $120 \mathrm{~nm}$ соответственно. Скорости звука в образцах различаются слабо, составляя $3.0 \pm 0.3 \mathrm{~km} / \mathrm{s}$. Значение скорости звука согласуется со значением скорости распространения продольных колебаний в кристаллах RDX [34]. Показатель поглощения в рамках использованного варианта оптоакустического метода определяется как отношение эффективной константы нарастания оптоакустического сигнала к скорости звука в среде. Полученные значения показателя поглощения композитов приведены на рис. 5. Характерная погрешность составляет 10\%, определяясь в основном точностью оценки скорости звука в конкретном образце.

\section{Методика расчета}

Моделирование поглощения электромагнитного излучения в композите проводилось в рамках одномерного уравнения переноса излучения в виде [15,23,35]

$$
\xi \frac{d I(x, \xi)}{d x}=-I(x, \xi)+\frac{\Lambda}{2} \int_{-1}^{1} I\left(x, \xi^{\prime}\right) \chi\left(\xi, \xi^{\prime}\right) d \xi^{\prime}
$$

где $x=\mu z$ - безразмерная координата, $\xi=\cos \theta-$ косинус сферического угла между нормалью к передней границе и направлением визирования, $I(\tau, \xi)$ - освещенность в точке $x$ в направлении $\xi, \Lambda=\mu_{\text {sca }} / \mu-$ альбедо однократного рассеяния света, $\chi(\xi)-$ фазовая функция рассеяния. Величины $\mu, \Lambda$ и функция $\chi(\xi)$ зависят от природы и концентрации центров рассеяния излучения в образце. Направление падающего излучения в соответствии с условиями эксперимента совпадало с нормалью к поверхности образца. Результаты решения уравнения (1) будут представлены в безразмерных единицах, что позволяет далее рассматривать падение на поверхность пучка единичной интенсивности.

Для решения уравнения (1) использовался метод сферических гармоник, заключающийся в разложении угловой составляющей освещенности по полиномам Лежандра $\left(P_{l}\right)$. Решение $I(x, \xi)$ разбивалось на диффузную $I_{s}(x, \xi)$ и коллимированную $I_{0}(x, \xi)$ составляющие: $I=I_{0}+I_{s}$, учитывающие излучение, поглотившееся в первом акте взаимодействия со средой и испытавшее не менее одного акта рассеяния соответственно. Коллимированная составляющая освещенности имеет вид [23]

$$
I_{0}=\left(1-R_{j}\right) \exp (-x) \delta(\xi-1),
$$

где $R_{f}-$ френелевский коэффициент отражения света, падающего перпендикулярно поверхности. Система уравнений для коэффициентов разложения $C_{m}(x)$ диффузной составляющей освещенности $I_{s}$ по полиномам Лежандра выглядит [23] следующим образом:

$$
\begin{aligned}
& \frac{1}{2 m+1}\left[(m+1) \frac{d C_{m+1}}{d \tau}+m \frac{d C_{m-1}}{d \tau}\right] \\
& +\left(1-\frac{\Lambda \chi_{m}}{2}\right) C_{m}=\left(1-R_{f}\right) \frac{\lambda \chi_{m}}{2} \exp (-x),
\end{aligned}
$$

где $\chi_{m}$ - коэффициенты разложения индикатрисы рассеяния по полиномам Лежандра.

Матричная запись френелевских граничных условий для системы уравнений (3) имеет вид [13-15,23]

$$
\sum_{m=0}^{N} R_{i m} C_{m}(0)=0,
$$

где матричные элементы определяются через френелевскую угловую зависимость энергетического коэффициента отражения $R(\xi)$ [13-15,23]:

$$
R_{i m}=\frac{2 m+1}{2} \int_{0}^{1} P_{m}(\xi)\left[1-(-1)^{m} R(\xi)\right] P_{l}(\xi) d \xi .
$$

Решение системы (3) можно представить [23] в виде

$$
C_{m}(x)=\sum_{l} a_{m l} \tilde{C}_{l} \exp \left(-\gamma_{l} x\right)+G_{m} \exp (-x),
$$

где суммирование ведется только по собственным векторам с положительными собственными значениями $\gamma_{l}$ [36]. Второе слагаемое в (5) представляет собой 


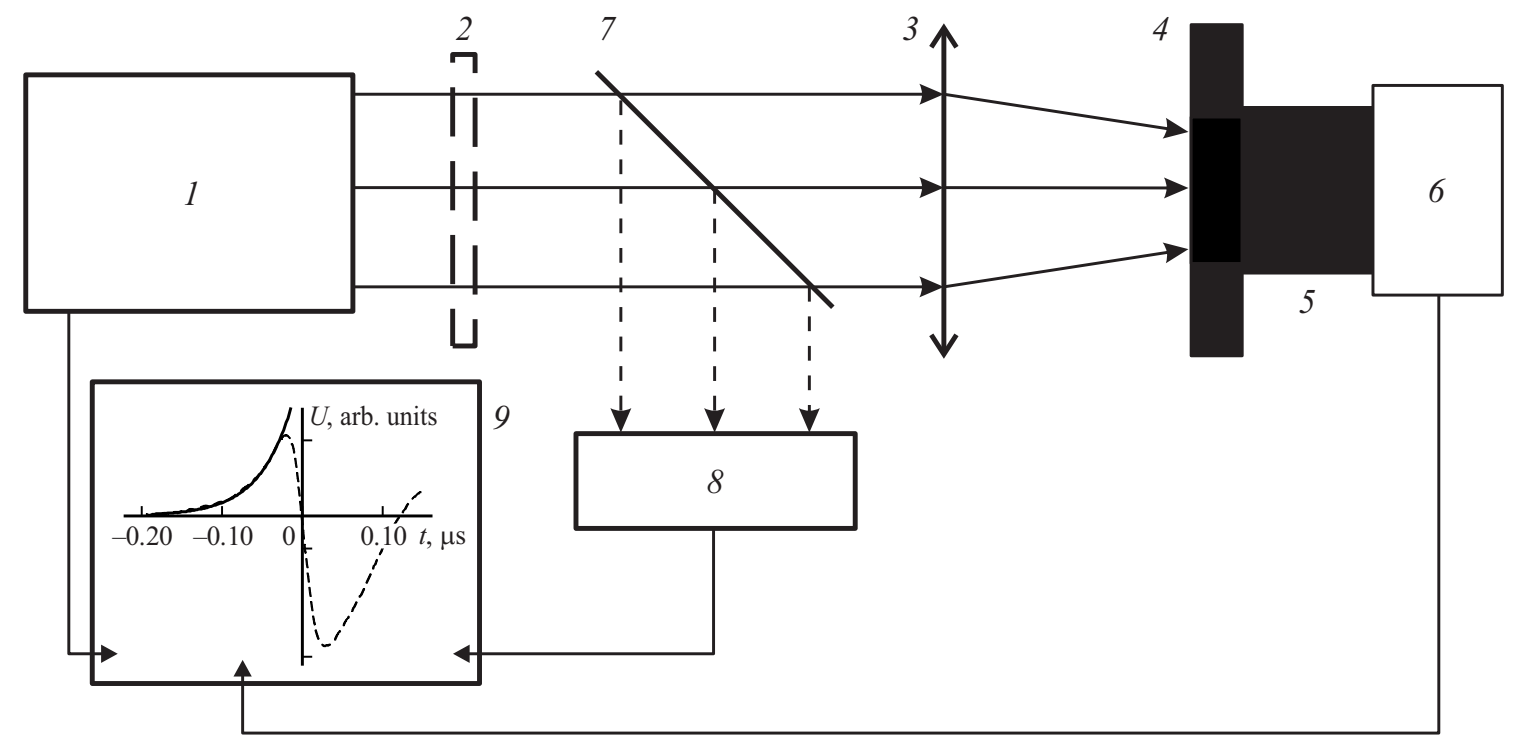

Рис. 3. Схема экспериментальной установки. 1 - YAG: $\mathrm{Nd}^{3+}$-лазер, 2 - нейтральные светофильтры, 3 - фокусирующая линза, 4 - образец в медном держателе, 5 - акустическая задержка, 6 - пьезопреобразователь, 7 - светоделительная пластина, 8 фотодиод, 9 - осциллограф.
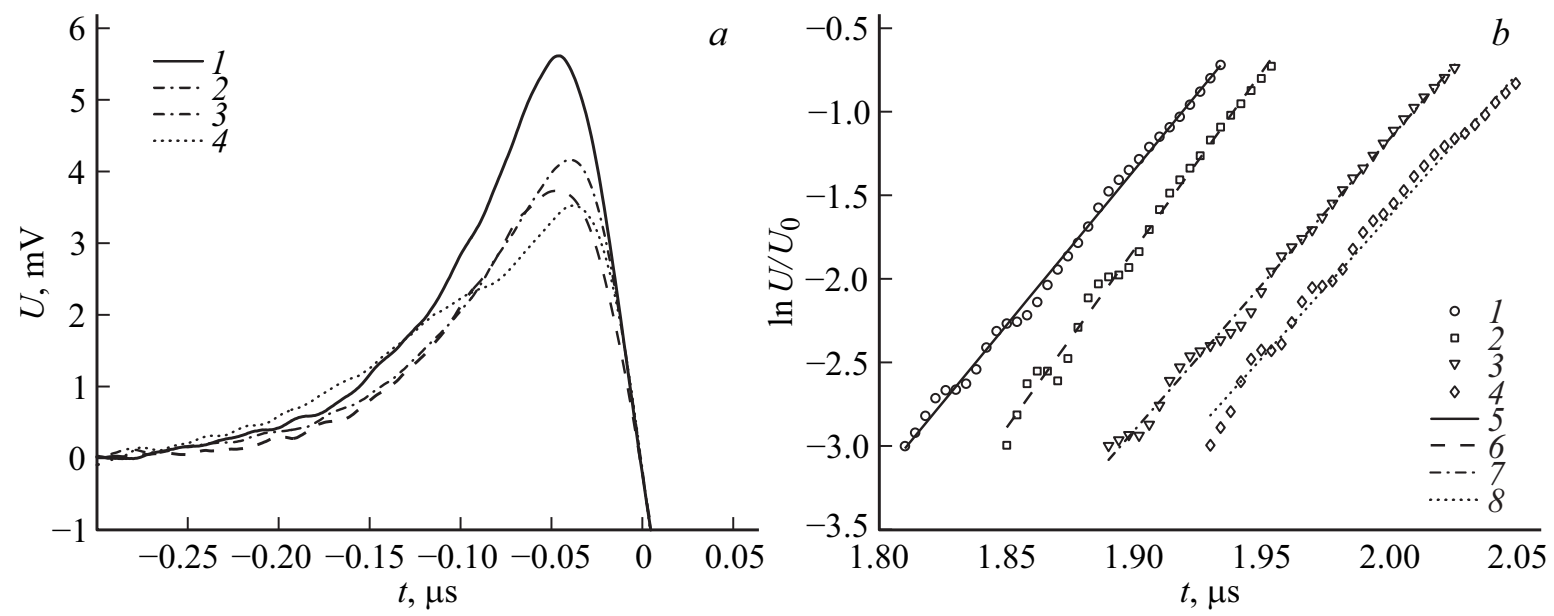

Рис. 4. (а) Экспериментальные оптоакустические сигналы в образцах RDX-ультрадисперсные частицы никеля со средними радиусами $40(1), 60(2), 80(3), 140 \mathrm{~nm}(4),(b)$ их начальные участки в полулогарифмических координатах вместе с аппроксимацией линейными зависимостями (5-8 соответственно) в зависимости от времени.

частное решение системы неоднородных уравнений (3) с коэффициентами

$$
G_{m}=-\left(1-R_{f}\right) \Lambda \sum_{m=0}^{N}\left[\delta_{p m}+A_{p m}\right]^{-1} B_{m},
$$

где используются обозначения

$$
\begin{aligned}
& A_{p m}=-\left[\frac{p+1}{2 p+1} \delta_{p, p+1}+\frac{p}{2 p+1} \delta_{p, p^{\prime}-1}\right]^{-1} \\
& \times {\left[\left(1-\frac{\Lambda \chi_{m}}{2}\right) \delta_{p^{\prime} m}\right], } \\
& B_{m}=\left[\frac{m+1}{2 m+1} \delta_{m, m^{\prime}+1}+\frac{m}{2 m+1} \delta_{m, m^{\prime}-1}\right]^{-1}\left[\frac{\chi_{m^{\prime}}}{2}\right] .
\end{aligned}
$$

Общее решение системы однородных уравнений, соответствующих (3), связано с первым слагаемым в правой части (5), которое записано в виде разложения по собственным векторам $a_{m l}$ с соответствующими собственными числами $\gamma_{l}$. Граничные условия (4) образуют переопределенную систему из $N+1$ уравнений, для решения которой использовался метод наименьших квадратов. В результате для коэффициентов $\tilde{C}_{l}$ получаем следующее уравнение, записанное в матричной форме [23]:

$$
\tilde{C}=-\left[(R a)^{T}(R a)\right]^{-1}(R a)^{T}(R G),
$$

где в матрице а оставлены только собственные векторы с положительными собственными значениями, между со- 


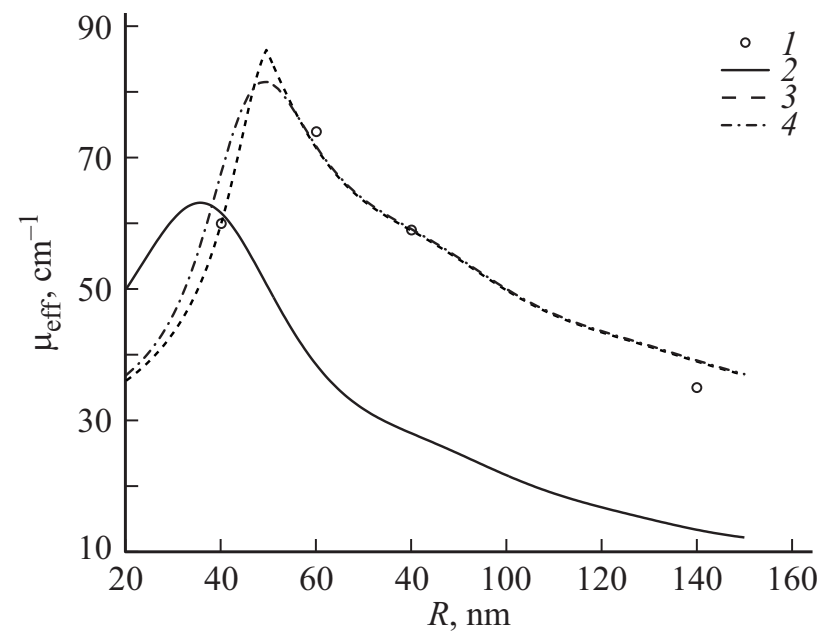

Рис. 5. Влияние радиуса наночастиц никеля на показатель поглощения композитов RDX-никель: 1 - экспериментальные данные, полученные оптоакустическим методом, 2 - расчет в пренебрежении порами, 3 - расчет с учетом пор радиуса $49.5 \mathrm{~nm}$ и концентрацией $1.3 \cdot 10^{13} \mathrm{~cm}^{-3}, 4$ - расчет при тех же значениях средней концентрации и радиусе пор с дисперсией $10 \mathrm{~nm}$.

множителями в (7) производится операция матричного умножения.

Коэффициенты разложения освещенности по полиномам Лежандра на границе $C_{m}(0)$ позволяют определить коэффициент отражения:

$$
\rho=\left(\sum_{m=0}^{N}(-1)^{m} C_{m}(0) \zeta_{m}+R_{f}\right),
$$

где

$$
\xi_{m}-\frac{2 m+1}{2} \int_{0}^{1} P_{m}(\xi)[1-R(\xi)] \xi d \xi .
$$

Распределение плотности поглощаемой мощности, нормированной на плотность мощности, падающей на поверхность, имеет вид

$$
A(z)=\mu(1-\Lambda)\left\lfloor C_{0}(z)+\left(1-R_{f}\right) \exp (-\mu z)\right\rfloor .
$$

Для расчета эффективного показателя поглощения $\mu_{\mathrm{eff}}$ зависимость $A(z)$ аппроксимировалась экспоненциальной функцией на участке от $5 \%$ до $50 \%$ амплитуды. Данная величина количественно характеризует профиль поглощения излучения с учетом его многократного рассеяния в образце.

\section{Моделирование зависимости $\mu_{\mathrm{eff}}(\boldsymbol{R})$}

Рассмотрим возможности интерпретации полученных зависимостей измеренного показателя поглощения от радиуса частиц. На рис. 6, $a$ приведены рассчитанные в рамках теории Ми зависимости оптических свойств ультрадисперсных частиц никеля на длине волны $532 \mathrm{~nm}$ (комплексный показатель преломления $1.7374-3.1490 i$ [37]) в матрице RDX (показатель преломления $1.594[14,38])$ от их радиуса. Наибольший коэффициент эффективности поглощения $\sigma_{\mathrm{abs}}(R) /\left(\pi R^{2}\right)$, равный 2.287, имеют частицы с радиусом $39 \mathrm{~nm}$, при этом коэффициент эффективности рассеяния составляет 1.203. Коэффициент эффективности рассеяния возрастает в области 25-50 nm, слабо отклоняясь от значения 2 при больших радиусах. Наибольший коэффициент эффективности ослабления проявляют частицы с радиусом $48 \mathrm{~nm}$. Фазовая функция рассеяния излучения для частиц с радиусом менее $60 \mathrm{~nm}$ слабо анизотропная.

На рис. 7, $a$ показаны рассчитанные распределения поглощенной энергии в образце при четырех значениях радиуса частиц. Распределения могут быть удовлетворительно описаны экспоненциальными функциями с эффективными показателями поглощения 61.6, 38.5, 28.0 и $13.3 \mathrm{~cm}^{-1}$ при радиусе частиц $40,60,80$ и $140 \mathrm{~nm}$ соответственно. Рассчитанная с использованием уравнения переноса излучения зависимость эффективного показателя поглощения от радиуса ультрадисперсных частиц никеля в матрице RDX приведена на рис. 5. Наибольший эффективный показатель поглощения наблюдается при радиусе частиц $37 \mathrm{~nm}$, что близко к положению максимума функции $\sigma_{\mathrm{abs}}(R) /\left(\pi R^{2}\right)$. В соответствии с результатами расчета следует ожидать уменьшение эффективного показателя поглощения композитов при росте радиуса частиц $(R>40 \mathrm{~nm})$, в то же время экспериментальная зависимость в данной области радиусов немонотонная.

Порошок RDX обладает плохой способностью к спрессовыванию. Прессованные таблетки RDX, не содержащие ультрадисперсных частиц металлов, непрозрачны в отличие от прессованных образцов PETN. Поры, находящиеся в объеме образца, способны не только приводить к увеличению показателя рассеяния [14], но и влиять на оптические свойства наночастиц при их попадании в поры $[15,39]$. На рис. $6, b$ приведены рассчитанные зависимости коэффициентов эффективности поглощения и рассеяния света с длиной волны $532 \mathrm{~nm}$ от радиуса частиц никеля в воздухе. Максимальный коэффициент эффективности поглощения 1.411 наблюдается в воздушной среде при радиусе частиц $72.3 \mathrm{~nm}$, а первый максимум на зависимости коэффициента эффективности рассеяния 2.357 наблюдается при радиусе частиц никеля $90.9 \mathrm{~nm}$.

Адаптируем методику моделирования оптических свойств композитов RDX-частицы алюминия с учетом пор [15] для расчета оптических свойств композитов $\mathrm{RDX}$-частицы никеля. Для расчета фазовой функции рассеяния, коэффициентов эффективности ослабления и рассеяния света на отдельных ультрадисперсных частицах и порах использовалась теория Ми $[1,40]$. Частица металла, находящаяся внутри поры, рассматривалась как двуслойная концентрическая сфера (металлическое ядро-воздушная оболочка). В этом случае для расчета оптических свойств применялась теория Адена- 

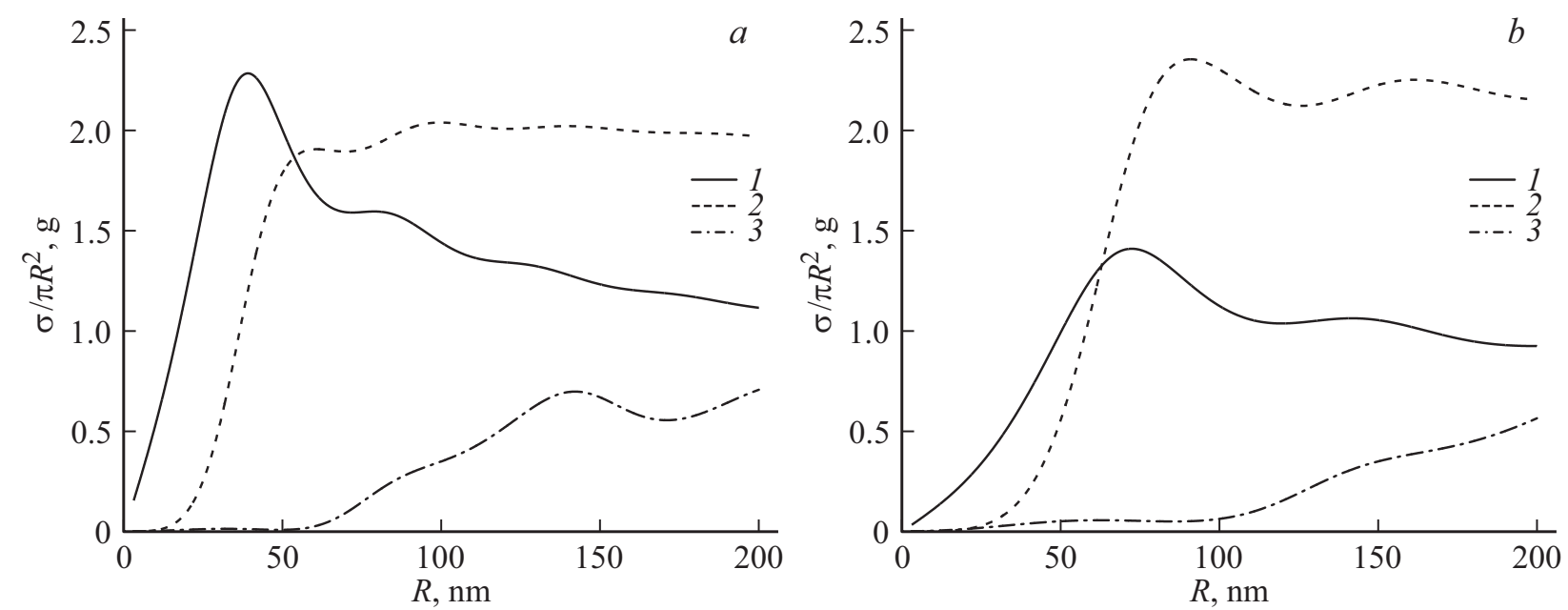

Рис. 6. Рассчитанные в рамках теории Ми зависимости коэффициентов эффективности поглощения (1) и рассеяния света (2) и модуль среднего косинуса угла рассеяния (3) излучения от радиуса наночастиц никеля в матрице $\mathrm{RDX}(a)$ и в воздухе $(b)$ при длине волны $532 \mathrm{~nm}$.
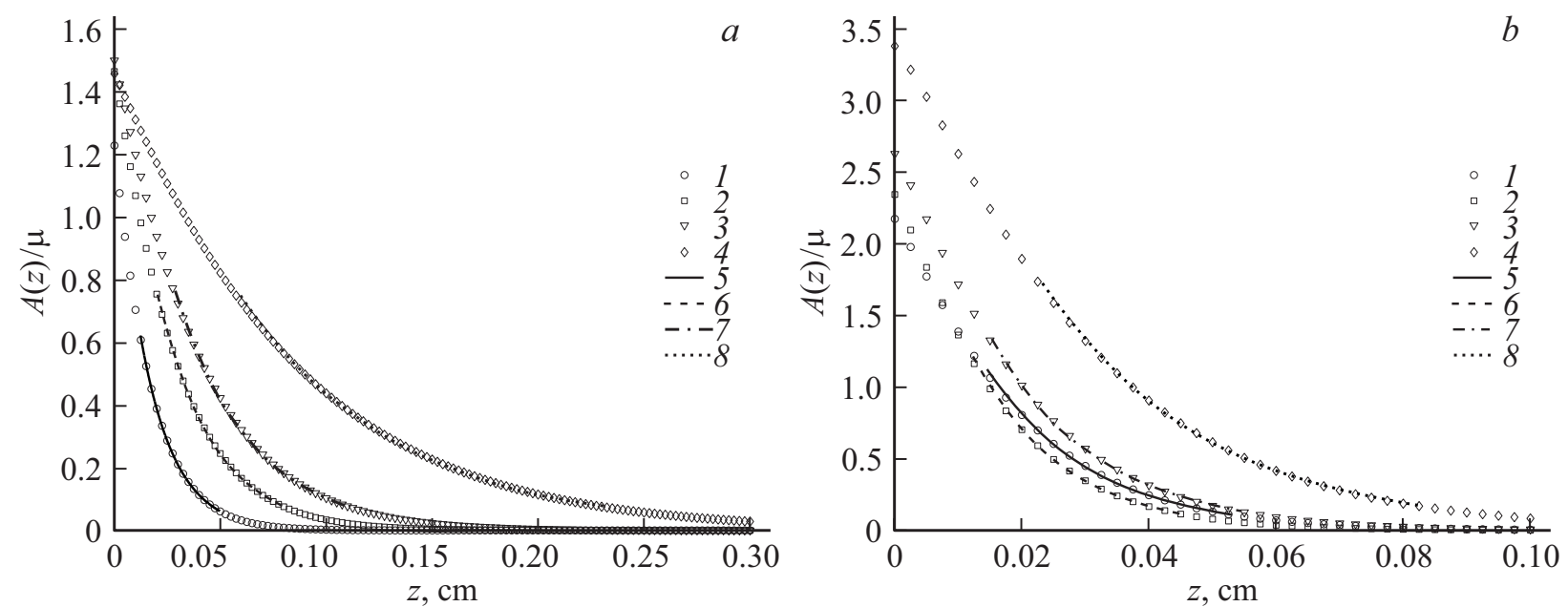

Рис. 7. Рассчитанные распределения поглощенной энергии в композите RDX-Ni при радиусе наночастиц 40 (1), 60 (2), 80 (3), $140(4) \mathrm{nm}$ без учета пор (a) и при наличии пор с радиусом $49.5 \mathrm{~nm}$ и концентрацией $1.3 \cdot 10^{13} \mathrm{~cm}^{-3}(b)$. Кривыми $5-8$ показано описание участка зависимости экспоненциальной функцией.

Керкера [41]. Данные подходы ранее апробированы при моделировании оптических свойств наночастиц с оболочкой пара [39] и композитов RDX-ультрадисперсные частицы алюминия $[14,15]$.

Предполагалось, что пористую структуру образца можно описать двумя параметрами: концентрацией пор и средним радиусом поры. Считалось, что в поре может разместиться только одна ультрадисперсная частица. Если концентрация частиц меньше, чем пор, дополнительно учитывалось рассеяние света на незаполненных порах. В противоположном случае все поры считались заполненными, и учитывалось поглощение и рассеяние света или на частицах металла в матрице RDX, или (в зависимости от соотношения радиусов металлической частицы и поры) на наночастицах металла с оболочкой поры в матрице RDX. Для расчета оптических свойств элементарного объема среды (показателей ослабления $\mu$ и рассеяния $\mu_{\text {sca }}$, а также фазовой функции рассеяния $\chi$ ) использовались выражения (10)-(12) соответственно:

$$
\mu= \begin{cases}n_{\mathrm{Ni}}+\sigma_{\mathrm{Ni}}+\left(n_{p}-n_{\mathrm{Ni}}\right) \sigma_{p}, & n_{p} \geq n_{\mathrm{Ni}}, R_{\mathrm{Ni}} \geq R_{p} ; \\ n_{\mathrm{Ni}}+\sigma_{\mathrm{Ni} p}+\left(n_{p}-n_{\mathrm{Ni}}\right) \sigma_{p}, & n_{p} \geq n_{\mathrm{Ni}}, R_{\mathrm{Ni}}<R_{p} ; \\ n_{p}+\sigma_{\mathrm{Ni} p}+\left(n_{\mathrm{Ni}}-n_{p}\right) \sigma_{\mathrm{Ni}}, & n_{p}<n_{\mathrm{Ni}}, R_{\mathrm{Ni}}<R_{p} ; \\ n_{\mathrm{Ni}} \sigma_{\mathrm{Ni}}, & n_{p}<n \mathrm{Ni}, R_{\mathrm{Ni}} \geq R_{p},\end{cases}
$$

$$
\mu_{\mathrm{sca}}= \begin{cases}n_{\mathrm{Ni}}+\sigma_{\mathrm{Ni}}^{\mathrm{sca}}+\left(n_{p}-n_{\mathrm{Ni}}\right) \sigma_{p}^{\mathrm{sca}}, & n_{p} \geq n_{\mathrm{Ni}}, R_{\mathrm{Ni}} \geq R_{p} ; \\ n_{\mathrm{Ni}}+\sigma_{\mathrm{Na} p}^{\mathrm{sca}}+\left(n_{p}-n_{\mathrm{Ni}}\right) \sigma_{p}^{\text {sca }}, & n_{p} \geq n_{\mathrm{Ni}}, R_{\mathrm{Ni}}<R_{p} ; \\ n_{p}+\sigma_{\mathrm{Ni} p}^{\mathrm{sca}}+\left(n_{\mathrm{Ni}}-n_{p}\right) \sigma_{\mathrm{Ni}}^{\text {sca }}, & n_{p}<n_{\mathrm{Ni}}, R_{\mathrm{Ni}}<R_{p} ; \\ n_{\mathrm{Ni}} \sigma_{\mathrm{Ni}}^{\text {sca }}, & n_{p}<n \mathrm{Ni}, R_{\mathrm{Ni}} \geq R_{p},\end{cases}
$$



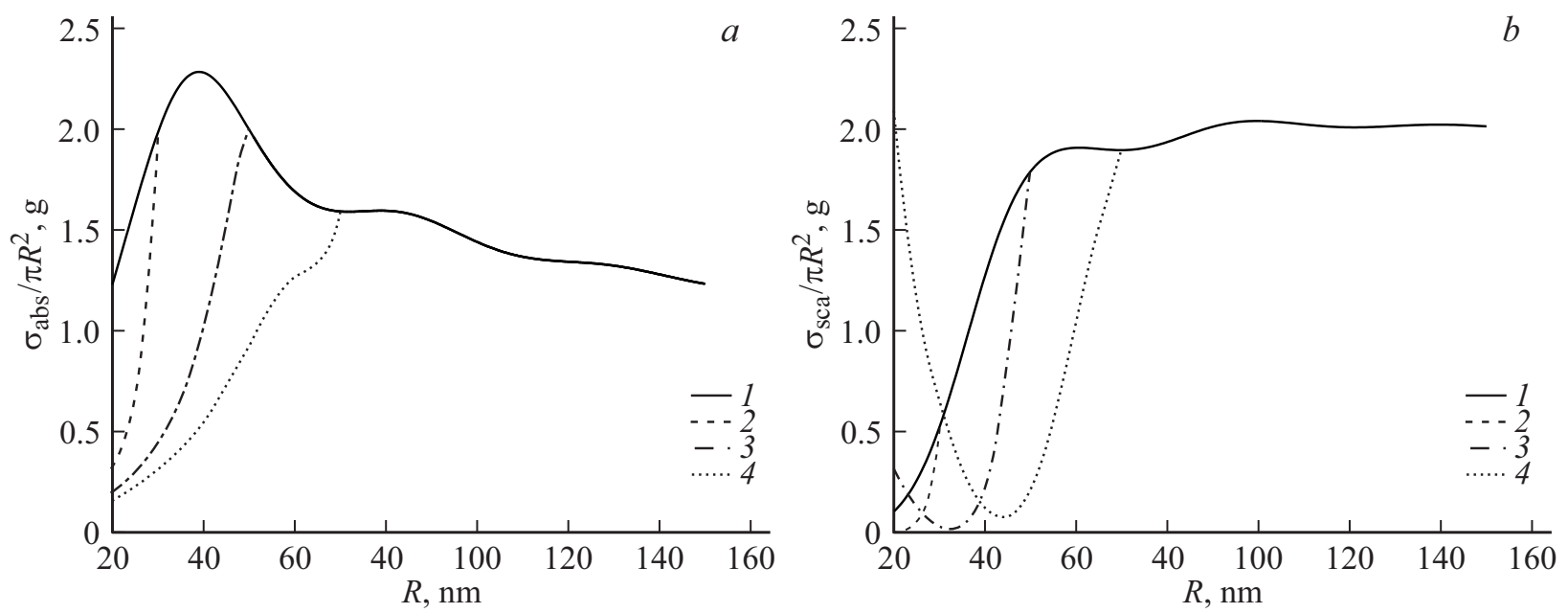

Рис. 8. Зависимости сечений поглощения $(a)$ и рассеяния $(b)$ излучения с длиной волны 532 nт наночастицами никеля от их радиуса в матрице RDX без пор (1) и при их локализации в порах с радиусом 30 (2), 50 (3), $70 \mathrm{~nm}(4)$.

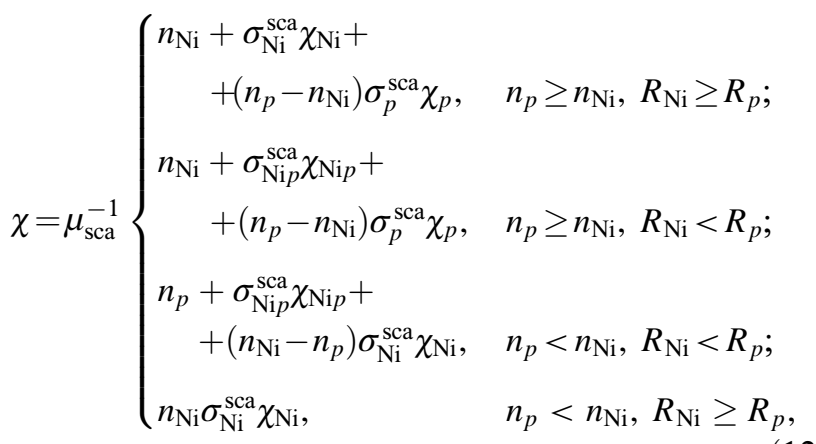

где $n_{p}$ и $n_{\mathrm{Ni}}$ - концентрации пор и частиц никеля $\left(\right.$ в $\left.\mathrm{cm}^{-3}\right)$ соответственно, $R_{\mathrm{Ni}}$ и $R_{p}$ - радиусы частиц никеля и пор соответственно, $\sigma, \sigma^{\text {sca }}$ и $\chi-$ сечение ослабления, сечение рассеяния излучения и нормированная фазовая функция рассеяния. Нижние индексы $\mathrm{Ni}$, $p$ и $\mathrm{Ni} / p$ указывают, что соответствующие величины относятся к частице никеля, поре и частице никеля внутри поры. В случае поры и частиц никеля вне пор (или в поре меньшего радиуса) расчет сечений и фазовой функции выполнялся в рамках теории Ми [40], в случае частиц внутри поры использовалась теория рассеяния излучения на двуслойной концентрической сфере Адена-Керкера [41].

При решении обратной задачи варьировался эффективный радиус пор $R_{p}$ и их концентрация $n_{p}$. С использованием выражений (10)-(12) рассчитывались показатели ослабления, рассеяния и фазовая функция рассеяния излучения в композите и распределение поглощенной плотности мощности по формулам (3)-(9). Далее определялся эффективный показатель поглощения. Сумма квадратов отклонений показателей поглощения, вычисленных и экспериментально определенных оптоакустическим методом, минимизировалась методом НейлдераМида.

Рассмотрим влияние пор на оптические свойства композита в рамках модели (1)-(12). Поры не приводят к каким-либо изменениям, если их радиус меньше радиуса частиц и концентрация также меньше, чем у наночастиц. Эта ситуация соответствует заполнению всех пор частицами. Если концентрация пор становится больше, чем концентрация частиц, но $R_{p}<R_{\mathrm{Ni}}$, поры приводят к появлению дополнительного вклада в показатель рассеяния и фактор анизотропии. Поскольку рассеяние на порах близко к релеевскому, рост их концентрации приведет к уменьшению среднего косинуса угла рассеяния в элементарном объеме вещества. Если радиус пор больше радиуса ультрадисперсных частиц, возникающая оболочка с малым показателем преломления будет приводить к уменьшению коэффициента эффективности поглощения света частицами металлов. В этом случае эффект будет неаддитивным.

Рассчитанные в рамках теории рассеяния света на двуслойной концентрической сфере зависимости сечений поглощения и рассеяния света частицами никеля, находящимися в порах с радиусами 30, 50 и $70 \mathrm{~nm}$, приведены на рис. $8, a$ и $8, b$ соответственно. При $R_{p}>R_{\mathrm{Ni}}$ поровая оболочка приводит к уменьшению сечения поглощения света частицей металла, поскольку воздух имеет пренебрежимо малую мнимую часть показателя преломления. Сечение рассеяния возрастает в области малых радиусов частиц при наличии поры. Сечение рассеяния света с длиной волны $532 \mathrm{~nm}$ на поре с радиусом $70 \mathrm{~nm}$ составляет $3 \cdot 10^{-11} \mathrm{~cm}^{-2}$. С учетом нормировки на геометрическое сечение частицы никеля отношение составит 2.39 при $R=20 \mathrm{~nm}$, что согласуется с рис. 8. На рис. 8 наблюдаются разрывы производной при $R_{p}=R_{\mathrm{Ni}}$, связанные с тем, что при больших радиусах поровая оболочка отсутствует.

На основании вышеизложенного возникает принципиальная возможность, варьируя средний радиус пор и их концентрацию, получить немонотонную зависимость эффективного показателя поглощения излучения с длиной волны $532 \mathrm{~nm}$ от радиуса ультрадисперсных ча- 
стиц $(R>40 \mathrm{~nm})$. Результаты решения обратной задачи приведены на рис. 5 (кривая 3), который иллюстрирует удовлетворительное описание данных эксперимента. Определенные значения варьируемых параметров составили $R_{p}=49.5 \mathrm{~nm}, C_{p}=1.3 \cdot 10^{13} \mathrm{~cm}^{-3}$. Распределения поглощенной энергии, рассчитанные с указанными радиусом и концентрацией пор, приведены на рис. $7, b$. Из рисунка также виден немонотонный характер зависимости $\mu_{\mathrm{eff}}(R)$, возникающий при наличии пор.

При радиусе частиц никеля $40 \mathrm{~nm}$ их концентрация (массовая доля $0.05 \%$ ) составляет $3.8 \cdot 10^{11} \mathrm{~cm}^{-3}$, а при $R_{\mathrm{Ni}}=120 \mathrm{~nm}-8.9 \cdot 10^{9} \mathrm{~cm}^{-3}$. То есть, неаддитивный случай реализуется только для наименьшего из использованных в эксперименте значений радиуса частиц. На рассчитанной зависимости $\mu_{\mathrm{eff}}(R)$ наблюдается разрыв производной в точке максимума, соответствующей равенству $R_{p}=R_{\mathrm{Ni}}$.

В работе [15] выполнено моделирование оптических свойств композитов RDX-наночастицы алюминия в приближении наночастиц одного радиуса и наличия релеевского распределения пор по размерам. Для оценки влияния распределения пор по размерам был выполнен расчет зависимости $\mu_{\mathrm{eff}}(R)$ в предположении, что последнее может быть описано функцией ошибок $\left(\sim \exp \left[-\left(R_{p}-R_{a}\right)^{2} / S^{2}\right]\right)$ при $S=10 \mathrm{~nm}$ (рис. 5). Из полученной зависимости следует, что учет распределения пор по размерам устраняет разрыв производной в точке максимума, в остальном интервале значений различия не существенны. Таким образом, дисперсия распределения пор по размерам является более слабым параметром, чем средний радиус пор и их концентрация, и пытаться оценить его можно только при близости среднего радиуса поры и радиуса частицы.

\section{Заключение}

Экспериментально определены показатели поглощения излучения с длиной волны $532 \mathrm{~nm}$ в композитных материалах RDX-ультрадисперсные частицы никеля лазерным оптоакустическим методом. Показано, что зависимость экспериментального показателя поглощения от радиуса частиц при постоянной массовой доле немонотонная. Для интерпретации результатов эксперимента использовалась теория переноса монохроматического излучения с расчетом оптических свойств наночастиц в рамках теории Ми и ее модификаций. Показано, что оптические свойства композита связаны с наличием в нем остаточной пористости со средним радиусом пор $49.5 \mathrm{~nm}$ и концентрацией $1.3 \cdot 10^{13} \mathrm{~cm}^{-3}$.

\section{Благодарность}

Авторы благодарят Р.П. Колмыкова за предоставленные ультрадисперсные порошки никеля и Д.М. Руссакова за их характеризацию методами электронной микроскопии.

\section{Финансирование работы}

Работа выполнена при поддержке РФФИ (проекты № 18-03-00421_a, № 19-33-90261), методическая часть в рамках гос. задания ИУХМ ФИЦ УУХ СО РАН (проект AАAА-A17-117041910150-2, руководитель Адуев Б.П.) на оборудовании КемЦКП ФИЦ УУХ СО РАН.

\section{Конфлликт интересов}

Авторы заявляют, что у них нет конфликта интересов.

\section{Список литературы}

[1] Klimov V.V. Nanoplazmonika. 2ed edition. Moscow: Fizmatlit, 2010. 480 р.; Климов В.В. Наноплазмоника. 2-е изд., испр. М.: ФИЗМАТЛИТ, 2010. 480 с. ISBN 978-5-9221-1205-5

[2] Фарафонов В.Г., Ильин В.Б., Прокопьева М.С., Тулегенов А.Р., Устимов В.И. // Опт. и спектр. 2019. Т. 126. № 4. C. 443; Farafonov V.G., Il'in V.B., Prokopjeva M.S., Tulegenov A.R., Ustimov V.I. // Opt. Spectrosc. 2019. V. 126. N 4. P. 360. doi 10.1134/S0030400X19040076

[3] Knight M.W., King N.S., Liu L., Everitt H.O., Nordlander P., Halas N.J. // ACS Nano. 2014. V. 8. P. 834-840. doi 10.1021/nn405495q

[4] Temple T.L., Bagnall D.M. // J. Appl. Phys. 2011. V. 109. P. 084343 . doi 10.1063/1.3574657

[5] Nikbakht M. // JQSRT. 2018. V. 221. P. 164. doi 10.1016/j.jqsrt.2018.10.005

[6] Rasskazov I.L., Karpov S.V., Markel V.A. // Phys. Rev. B. 2014. V. 90. N 7. P. 075405. doi 10.1103/PhysRevB.90.075405

[7] Рассказов И.Л., Маркель В.А., Карпов С.В. // Опт. и спектр. 2013. 115. № 5. С. 753; Rasskazov I.L., Markel V.A., Karpov S.V. // Opt. Spectrosc. 2013. V. 115. N 5. P. 666. doi 10.1134/S0030400X13110180

[8] Markel V.A., Sarychev A.K. // Phys. Rev. B. 2007. V. 75. N 8. P. 085426. doi 10.1103/PhysRevB.75.085426

[9] Khanadeev V.A., Khlebtsov B.N., Khlebtsov N.G. // JQSRT. 2017. V. 187. P. 1. doi 10.1016/j.jqsrt.2016.09.004

[10] Zhaolong Wang, Xiaojun Quan, Zhuomin Zhang, Ping Cheng // JQSRT. 2018. V. 205. P. 291. doi $0.1016 /$ j.jqsrt.2017.08.001

[11] Jaiswal J., Mourya S., Malik G., Chandra R. // JOSA. A. 2018. V. 35. N 5. P. 740. doi 10.1364/JOSAA.35.000740

[12] Khlebtsov B.N., Khanadeev V.A., Panfilova E.V., Inozemtseva O.A., Burov A.M., Khlebtsov N.G. // JQSRT. 2013. V. 121. P. 23. doi 10.1016/j.jqsrt.2013.03.001

[13] Адуев Б.П., Нурмухаметов Д.Р., Белокуров Г.М., Звеков А.А., Каленский А.В., Никитин А.П., Лисков И.Ю. // ЖТФ. 2014. Т. 84. № 9. С. 126. Aduev B.P., Nurmukhametov D.R., Belokurov G.M., Zvekov A.A., Nikitin A.P., Liskov I.Y., Kalenskii A.V. // Technical Physics. The Russ. J. Appl. Phys. 2014. V. 59. N 9. P. 1387. doi 10.1134/S1063784214090023

[14] Адуев Б.П., Белокуров Г.М., Нурмухаметов Д.Р., Лисков И.Ю., Нелюбина Н.В., Звеков А.А., Каленский А.В. // Опт. и спектр. 2018. Т. 125. № 5. С. 600; Aduev B.P., Belokurov G.M., Nurmukhametov D.R., Liskov I.Yu., Nelyubina N.V., Zvekov A.A., Kalenskii A.V. // Opt. Spectrosc. 2018. V. 125. N 5. P. 632. doi 10.1134/S0030400X18110024 
[15] Звеков А.А., Ананьева М.В., Каленский А.В., Адуев Б.П., Галкина E.B. // ЖПС. 2019. Т. 86. № 3. С. 438; Zvekov A.A., Anan'eva M.V., Kalenskii A.V., Aduev B.P., Galkina E.V. // J. Appl. Spectrosc. 2019. V. 86. N 3. P. 470. doi 10.1007/s10812019-00843-z

[16] Ананьева М.В., Нурмухаметов Д.Р., Зверев А.С., Нелюбина Н.В., Звеков А.А., Руссаков Д.М., Каленский А.В., Еременко А.Н. // Известия вузов. Физика. 2017. Т. 60. № 10. C. 3; Anan'eva M.V., Zverev A.S., Russakov D.M., Kalenskii A.V., Nurmukhametov D.R., Nelyubina N.V., Zvekov A.A., Eremenko A.N. // Russian Physics J. 2018. V. 60. N 10. P. 1651. doi 10.1007/s11182-018-1264-2

[17] Каленский А.В., Звеков А.А., Галкина Е.В., Нурмухаметов Д.Р. // Компьютерная оптика. 2018. Т. 42. № 2. C. 254; Kalenskii A.V., Zvekov A.A., Galkina E.V., Nurmuhametov D.R. // Computer Optics. 2018. V. 42. N 2. P. 254. doi 10.18287/2412-6179-2018-42-2-254-262

[18] Xiaoyu D., Haussener S. // JQSRT. 2018. V. 206. P. 378. doi 10.1016/j.jqsrt.2017.11.025

[19] Aréstegui O.S., Poma P.Y.N., Herculano L.S., Lukasievicz G.V.B., Guimar aes F.B., Malacarne L.C., Baesso M.L., Bialkowski S.E., Astrath N.G.C. // Appl. Spectrosc. 2014. V. 68. N 7. P. 777. doi 10.1366/13-07404

[20] Гусев В.Э., Карабутов А.А. Лазерная оптоакустика. М.: Наука, 1991. 304 с.; Gusev V.E., Karabutov A.A. Laser Optoacoustics. N.Y: AIP, 1993. 304 p.

[21] Moreno T.V., Filho N.E.S., Novatski A., Malacarne L.C., Dias G.S., Volnistem E.A., Astrath N.G.C. // Appl. Spectrosc. 2018. V. 72. N 2. P. 251. doi $10.1177 / 0003702817727729$

[22] Nikitin A.P., Zvekov A.A., Kalenskii A.V., Ananeva M.V., Nurmukhametov D.R. // Thermal science. 2019. V. 23. N 2. P. 553-560. doi 10.2298/TSCI19S2553N

[23] Ананьева М.В., Звеков А.А., Каленский А.В. // Письма в ЖТФ. 2019. Т. 45. № 7. С. 45; Anan'eva M.V., Zvekov A.A., Kalenskii A.V. // Technical Phys. Lett. 2019. V. 45. N 4. P. 352. doi 10.1134/S1063785019040035

[24] Адуев Б.П., Белокуров Г.М., Нурмухаметов Д.Р., Нелюбина Н.В. // Физика горения и взрыва. 2012. Т. 48. № 3. C. 127; Aduev B.P., Belokurov G.M., Nurmukhametov D.R., Nelyubinaa N.V. // Combustion, Explosion, and Shock Waves. 2012. V. 48. N 3. P. 361. doi 10.1134/S001050821203015X

[25] Адуев Б.П., Нурмухаметов Д.Р., Фурега Р.И., Звеков А.А. // Химическая физика. 2014. Т. 33. № 6. С. 37; Aduev B.P., Nurmukhametov D.R., Furega R.I., Zvekov A.A. // Russian J. Phys. Chem. B. 2014. V. 8. N 3. P. 352. doi $10.1134 / \mathrm{S} 1990793114030178$

[26] Каленский А.В., Звеков А.А., Никитин А.П., Ананьева М.В., Адуев Б.П. // Опт. и спектр. 2015. Т. 118. № 6. C. 1012; Kalenskii A.V., Zvekov A.A., Nikitin A.P., Ananeva M.V., Aduev B.P. // Opt. Spectrosc. 2015. V. 118. N 6. P. 978. doi 10.1134/S0030400X15060119

[27] Dumitra D.C.s, Dutu D.C., Matei C., Magureanu A.M., Petrus M., Popa C. // J. Optoelectron. Adv. Mater. 2007. V. 9. N 12. P. 3655.

[28] Santiesteban D.Y., Dumani D.S., Profili D., Emelianov S.Y. // Nano Lett. 2017. V. 17. N 10. P. 5984. doi 10.1021/acs.nanolett.7b02105

[29] Адуев Б.П., Нурмухаметов Д.Р., Белокуров Г.М., Фурега Р.И. // Физика горения и взрыва. 2015. Т. 51. № 3. C. 70; Aduev B.P., Nurmukhametov D.R., Belokurov G.M., Furega R.I. // Combustion, Explosion, and Shock Waves. 2015. V. 51. N 3. P. 347. doi 10.1134/S0010508215030107
[30] Nadal J.M., dos Anjos Camargo G., Novatski A., Macenhan W.R., Dias D.T., Barboza F.M., Lyra A., Roik J.R., de Paula J.P., Somer A., Farago P.V. // PLOS ONE. 2019. V. 14. N 3. e0213625. doi 10.1371/journal.pone.0213625

[31] Jun Xia, Junjie Yao, Lihong V. Wang. Photoacoustic Tomography: Principles and Advances // Progress In Electromagnetics Research. 2014. V. 147. P. 1. doi 10.2528/PIER 14032303

[32] Адуев Б.П., Нурмухаметов Д.Р., Колмыков Р.П., Никитин А.П., Ананьева М.В., Звеков А.А., Каленский А.В. // Химическая физика. 2016. Т. 35. № 8. C. 37; Aduev B.P., Nurmukhametov D.R., Kolmykov R.P., Nikitin A.P., Ananeva M.V., Zvekov A.A., Kalenskii A.V. // Russian J. Phys. Chem. B. 2016. V. 10. N 4. P. 621. doi $10.1134 / \mathrm{S} 1990793116040187$

[33] Карабутов А.А., Пеливанов И.М., Подымова Н.Б., Скипетров С.E. // Квант. электрон. 1999. Т. 29. № 3. C. 215; Karabutov A.A., Pelivanov I.M., Podymova N.B., Skipetrov S.E. // Quant. Electron. 1999. V. 29. P. 1054. doi 10.1070/QE1999v029n12ABEH001630

[34] Haycraft J.J., Stevens L.L., Eckhardt C.J. // J. Chem. Phys. 2006. V. 124. 024712. doi 10.1063/1.2141958

[35] Ishimaru A. Wave Propagation and Scattering in Random Media. Wiley-IEEE Press, 1999. 600 р. Перевод: Исимару А. Распространение и рассеяние волн в случайнонеоднородных средах. Т. 1. М.: Мир, 1981. С. 176.

[36] Panasyuk G.Y., Schotland J.C., Markel V.A. // J. Phys. A. 2006. V. 39. N 1. P. 115. doi 10.1088/0305-4470/39/1/009

[37] Palik E.D. Handbook of Optical Constants of Solids. San Diego: Academic Press Inc., 1985. V. 1. P. 405.

[38] Isbell R.A., Brewster M.Q. // Propellants, Explosives, Pyrotechnics. 1998. V. 23. N 4. P. 218. doi 10.1002/(SICI)15214087(199808)23:4<218::AID-PREP218>3.0.CO;2-A

[39] Pustovalov V.K., Astafyeva L.G. // Laser Phys. 2011. V. 21. N 12. P. 2098.

[40] Шифрин К.С. Рассеяние света в мутной среде. М.; Л.: Гос. изд. технико-теоретической литературы, 1951. 288 с. Shifrin K.S. Scattering of Light in Turbid Medium. Moscow: Gos.izd. techniko-teoreticheskoi literaturi, 1951. 288 p.

[41] Aden A.L., Kerker M. // J. Appl. Phys. 1951. V. 22. N 10. P. 1242. doi 10.1063/1.1699834 